\title{
Soluble total antigen derived from Toxoplasma gondii RH strain prevents apoptosis, but induces anti-apoptosis in human monocyte cell line
}

\author{
Hanieh Mohammad Rahimi ${ }^{1}$, Sara Nemati ${ }^{1}$, Helia Alavifard $^{2}$, Kaveh Baghaei ${ }^{2}$, Hamed Mirjalali $^{1}{ }^{(\mathbb{D}}$ and \\ Mohammad Reza Zali
}

\begin{abstract}
${ }^{1}$ Foodborne and Waterborne Diseases Research Centre, Research Institute for Gastroenterology and Liver Diseases, Shahid Beheshti University of Medical Sciences, Tehran, Iran;

${ }^{2}$ Basic and Molecular Epidemiology of Gastrointestinal Disorders Research Centre, Research Institute for Gastroenterology and Liver Diseases, Shahid Beheshti University of Medical Sciences, Tehran, Iran;

${ }^{3}$ Gastroenterology and Liver Diseases Research Centre, Research Institute for Gastroenterology and Liver Diseases, Shahid Beheshti University of Medical Sciences, Tehran, Iran
\end{abstract}

\begin{abstract}
Apoptosis plays crucial role in the pathogenesis of toxoplasmosis, as it limits further development of the disease. The current study aimed to investigate the effects of different concentrations of soluble total antigen (STAg) of Toxoplasma gondii (Nicolle et Manceaux, 1908) on the apoptotic and anti-apoptotic pathways. PMA-activated THP-1 cell line was sensed by T. gondii STAg and the expression patterns of caspase-3, -7, -8, -9, Bax, Bcl-2, and Mcl-1 genes were evaluated. The results showed statistically significant concentration-dependent overexpression of both Bcl-2 $(P$-value $<0.0001)$ and Mcl-1 $(P$-value $=0.0147)$. The cas-7 showed overexpression in all concentrations $(P$-value $<0.0001)$. The cas-3 was suppressed in concentrations 100,80 , and $40 \mu \mathrm{g}$, but statistically significant downregulated in concentrations 10 and $20 \mu \mathrm{g}$. The Bax was suppressed in concentrations 100 to $20 \mu \mathrm{g}$, while it slightly downregulated 1.42 fold $(P$-value $=0.0029)$ in concentration $10 \mu \mathrm{g}$. The expression of cas- 8 and -9 was suppressed in all concentrations. Our results indicated that $T$. gondii STAg downregulated and suppressed apoptotic and upregulated anti-apoptotic pathways. The upregulation of cas-7 in this study may indicate the role of $T$. gondii STAg in activation of inflammatory responses.
\end{abstract}

Keyword: Toxoplasma gondii, Soluble total antigen, Apoptosis, Mcl-1, Bcl-2.

Toxoplasmosis is a parasitic disease caused by Toxoplasma gondii (Nicolle et Manceaux, 1908), which usually causes no obvious symptoms in adults (Pappas et al. 2009). Toxoplasma gondii can infect all mammals; however, only felids are definitive hosts (Tenter et al. 2000). It is a cosmopolitan protozoan parasite, infecting more than one billion people of which most of them have chronic asymptomatic form (Aguirre et al. 2019). In individuals who have compromised immune systems, toxoplasmosis may lead to serious health problems, severe symptoms, and eventually death (Xiao and Yolken 2015). Infection during the pregnancy may affect offspring, which is known as congenital toxoplasmosis (Lourido 2019).

Apoptosis is the physiological cell death, which leads to elimination of unwanted or useless cells (D'Arcy 2019). This process plays a crucial role in the pathogenesis of toxoplasmosis, as well. During toxoplasmosis, apoptosis can play as a double edged sward (Mammari et al. 2019). Although this phenomenon may lead to cell death and limits further development of toxoplasmosis, $T$. gondii can use apoptosis to suppress the immune responses (Lüder et al. 2001). Ultrastructure evidence indicated that during the infection with $T$. gondii, tachyzoites were found in the cytosol of HeLa cells 10 min after infection and started to replicate $2-5 \mathrm{~h}$ after the infection (Toulah et al. 2011). After the entry, $T$. gondii modulates cell survival and cell death cycles to dysregulate the immune responses, which leads to unrestricted parasitemia and destruction of host tissues (Opferman and Korsmeyer 2003, Payne et al. 2003, Mammari et al. 2019).

In mammalian cells, there are at least two main pathways that lead to apoptosis, i.e., intrinsic pathway (mitochondria-mediated events) and extrinsic pathway (death receptor-mediated events) (Elmore 2007, Obeng 2021). B-cell lymphoma (Bcl) 2 family proteins, cytochrome $\mathrm{C}$, adaptor proteins, and caspases are the components of intrinsic pathway. The intrinsic pathway is stimulated by producing intracellular signals such as radiation (DNA

*Address for correspondence: Hamed Mirjalali, Foodborne and Waterborne Diseases Research Center, Research Institute for Gastroenterology and Liver Diseases, Shahid Beheshti University of Medical Sciences, Arabi Street, 1985717413, Chamran Highway, Tehran, Iran. Email: hamedmirjalali@ sbmu.ac.ir, hamed_mirjalali@hotmail.com 
damage), absence of certain growth factors, hormones, and cytokines. These stimuli cause changes in the mitochondrial outer membrane permeabilisation (MOMP) and release of cytochrome c (pro-apoptotic proteins) from the intermembrane space into the cytosol. In addition, caspase (cas)-9 activation leads to cas-3 activation and cell death (Green et al. 2004, Jin and El-Deiry 2005, Akhtar and Bokhari 2021).

Death receptors and ligands, adaptor proteins, and caspases are the components of extrinsic pathway. Until now, 14 caspases are known in mammals of which ten major caspases have been identified and broadly categorised into signaling/initiator (2, 8, 9, and 10), effector/executioner $(3,6$, and 7$)$, and inflammatory caspases $(1,4$, and 5) (Boatright et al. 2003, Yanumula and Cusick 2021).

The regulation and control of apoptotic mitochondrial events occur through members of the Bcl-2 family. Bcl2 family are key regulators in the molecular mechanisms of apoptosis. This family includes anti-apoptotic proteins, such as Bcl-2, Bcl-x, Bcl-XL, and Bcl-w, and pro-apoptotic proteins, i.e., Bax, Bak, Bid, Bad, Bim, and Bik. These proteins either are free in cytosol or attached to cell membrane. Bcl-2 family regulates the release of cytochrome $\mathrm{C}$ from the mitochondria via alteration of mitochondrial membrane permeability (Cheng et al. 2001, Akhtar and Bokhari 2021).

The exact effect of $T$. gondii or its antigens on the apoptotic and anti-apoptotic pathways are still unknown and previous study revealed controversial results (Goebel et al. 2001, Hwang et al. 2010, Li et al. 2016). In the current study, the effects of different concentrations of soluble total antigen (STAg) of T. gondii tachyzoites on the apoptotic and anti-apoptotic pathways were evaluated on human monocytes cell line.

\section{MATERIALS AND METHODS}

\section{Parasite preparation}

To provide sufficient number of Toxoplasma gondii, $1 \times 10^{5}$ tachyzoites of the virulent $\mathrm{RH}$ strain were inoculated to the peritoneal cavity of white laboratory mice, and were harvested from infected mice after 3-7 day of inoculation. Briefly, peritoneal fluid of infected mice was aspirated and centrifuged at $10,000 \mathrm{~g}$ for $10 \mathrm{~min}$. Supernatant was discarded and the pellet containing T. gondii tachyzoites were washed twice in phosphate-buffered saline (PBS; $\mathrm{pH}=7.4$ ). To prepare STAg, tachyzoites were suspended in $1 \mathrm{~mL}$ of lysis buffer ( $1 \%$ sodium dodecyl sulfate and 5mM EDTA) and sonicated (30 pulses for three times and 1 min intervals) on ice as described by Pazoki et al. (2021). After centrifugation for $10 \mathrm{~min}$ at $10,000 \mathrm{~g}$, total protein concentration was measured using the Bicinchoninic acid (BCA) assay (Parstous, Mashhad, Iran).

\section{THP-1 cell line cultivation}

The human monocyte THP-1 cell line was propagated in 1640 medium (RPMI 1640; Biosera, Arya Tous, Tehran, Iran) supplemented with $10 \%(\mathrm{v} / \mathrm{v})$ fetal bovine serum (FBS; Gibco, Thermo Fisher Scientific, Massachusetts, USA), 1\% penicillin/streptomycin (Thermo Fisher Scientific) at $37^{\circ} \mathrm{C}$ and $5 \% \mathrm{CO}_{2}$. THP-1 cells were counted by hemocytometer slide and $5 \times 10^{5}$ of cells were seeded in each well of a 12-well cell culture plate. To differentiate monocytes to macrophage M0, $30 \mathrm{ng} / \mathrm{ml}$ of phorbol 12-myristate 13-acetate (PMA; Santa Cruz Biotechnology Cat. No. sc-3576) were added to the cells. The plates were incubated for $36 \mathrm{~h}$ at $37^{\circ}$ $\mathrm{C}$ in $5 \% \mathrm{CO}_{2}$. Prior to the experiments and after morphological confirmation, supernatant medium containing non-adherent cells was discarded and replaced with PMA- and LPS-free cell culture medium (RPMI 1640 medium with 10\% FBS and 1\% antibiotic). M0 macrophages were rested for $24 \mathrm{~h}$ prior to experiments.

\section{Treatment of THP-1 cell line with different concentra- tions of Toxoplasma gondii STAg}

THP-1 cells were treated with 100, 80, 40, 20, and $10 \mu \mathrm{g} / \mathrm{ml}$ STAg. A well was treated with $20 \mathrm{ng} / \mathrm{ml}$ LPS (Santa Cruz Biotechnology Cat No. sc-3535) to compare its induction pattern with T. gondii STAg. A well full of PMA-activated THP-1 cell without any treatment either by $T$. gondii STAg or LPS, was considered as the control group. All groups were duplicate and evaluated $18 \mathrm{~h}$ after exposure.

\section{RNA isolation, cDNA synthesis, and Real-time RT-PCR}

Total RNA was extracted from THP-1 cells by the total RNA extraction kit (Yekta Tajhiz Azma, Tehran, Iran) according to the manufacturer's protocol. DNase (Thermo Fisher Scientific TM) treatment was performed to remove the probable residual DNA and to improve the quality of the extracted RNA according to the manufacturer's protocol. The RNA solution was finally collected and stored at $-70^{\circ} \mathrm{C}$ until cDNA synthesis. Prior to cDNA synthesis, the concentration of purified RNAs was determined by NanoDrop (NanoDrop Technologies, Wilmington, Delaware, USA) and RNA adjustment (normalisation) was performed. cDNA was constructed using cDNA synthesis kit (Yekta Tajhiz Azma, Tehran, Iran) according to the manufacturer's protocol.

To study the expression levels of the cas-3, 7, 8, 9, Bax, Bcl2, and Mcl-1, qRT-PCR using specific primers (Table1) was employed. Rotor-Gene Q (Qiagen, Hilden, Germany) thermocycler was employed to amplify targeted cDNAs in a 20 reaction mixture containing $10 \mu \mathrm{l} \mathrm{SYBR}$ Green qPCR Master Mix 2X (Ampliqon, Odense, Denmark), $5 \mu \mathrm{M}$ of primers. The standard amplification program was initial denaturation $95{ }^{\circ} \mathrm{C}$ for 10 min followed by 40 cycle of denaturation at $95^{\circ} \mathrm{C}$ for $20 \mathrm{~s}$, annealing at $59-61{ }^{\circ} \mathrm{C}$ for $30 \mathrm{~s}$, and extension at $72{ }^{\circ} \mathrm{C}$ for $20 \mathrm{~s}$. Melt curve analysis was performed to determine the specificity of each reaction. Reactions were carried out in duplicate and the transcriptional level of each gene was treated with $\beta$-actin as housekeeping gene. The relative expression level of each gene was analysed using the $2^{-\Delta \Delta C t}$ method and relative expression software tool (REST).

\section{Statistical analysis}

ANOVA test was applied to analyse the protein fold expression at different concentrations. Student's t-test was applied to analyse the real-time PCR data. $P$-value $<0.05$ was considered statistically significant. Statistical analysis was performed using GraphPad Prism software version 8.3.0.538.

\section{RESULTS}

The results of real-time PCR revealed increased expression of Bcl-2 and Mcl-1, as anti-apoptotic factors, in 

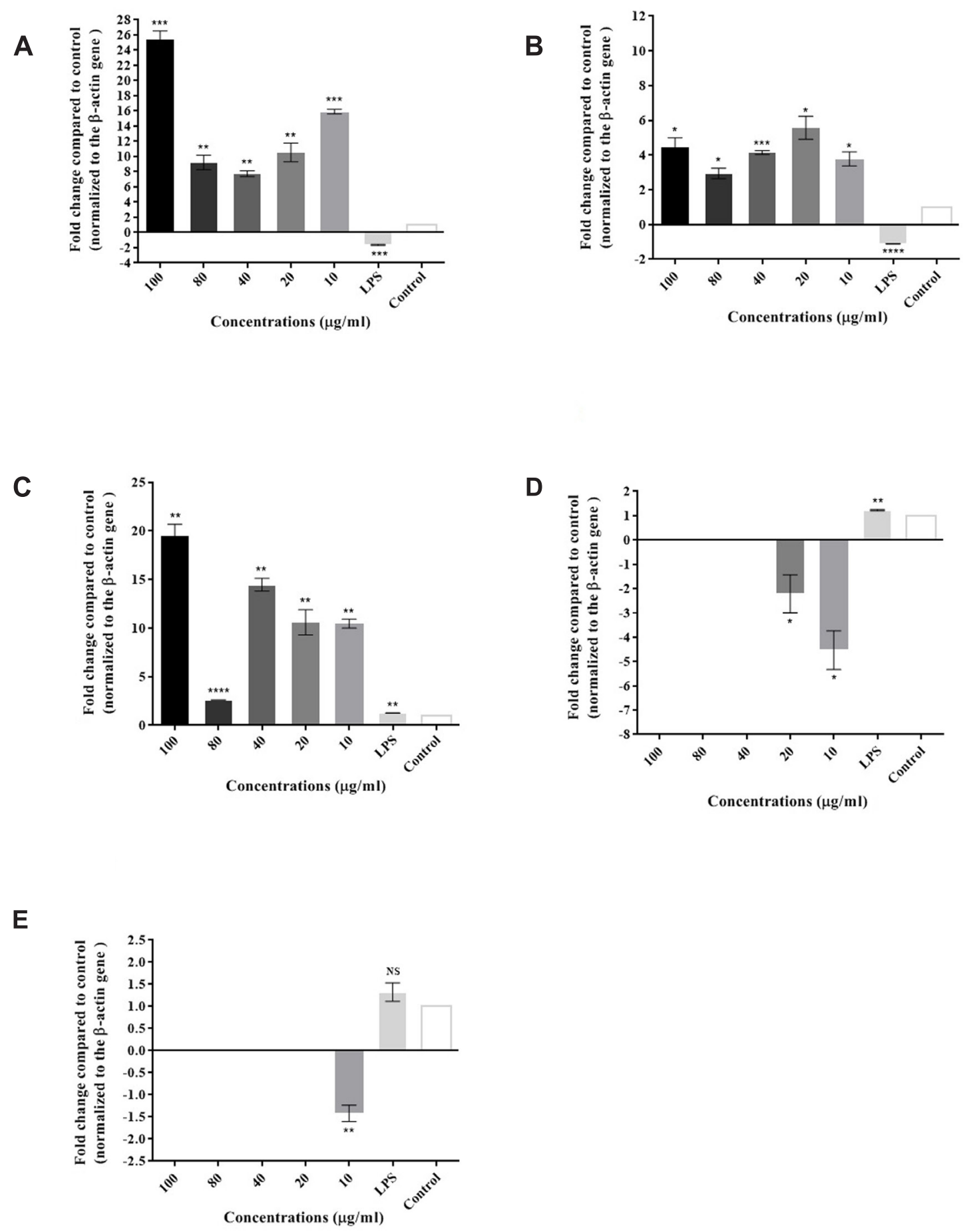

Fig 1. The comparison of the expression levels of Bcl-2 (A), Mcl-1 (B), Cas-7 (C), Cas-3 (D), and Bax (E) in THP-1 cell line regarding different concentrations of the soluble total antigen of Toxoplasma gondii (Nicolle et Manceaux, 1908) STAg. NS: not significant; * $P$ value $<0.05 ; * *$ value $<0.01 ; * * * P$ value $<0.001$

all concentrations of T. gondii STAg. Regarding the concentrations, the highest expression level in Bcl-2 was seen in $100 \mu \mathrm{g} / \mathrm{ml}$ of $T$. gondii STAg $(25.55$ fold; $P$-value $=$ $0.0008)$. In addition, a different expression was observed in Bcl-2 mRNA in other concentrations. Except concentration $100 \mu \mathrm{g}$, the Bcl-2 showed a statistically significant overexpression from $80 \mu \mathrm{g} / \mathrm{ml}$ to $10 \mu \mathrm{g} / \mathrm{ml}$ concentrations of $T$. gondii STAg $(P$-value $<0.0001)$ (Fig 1A).
Mcl-1 mRNA statistically overexpressed after exposure with $T$. gondii STAg in all concentrations $(P$-value $=$ $0.0147)$. The highest expression was seen in concentration $20 \mu \mathrm{g} / \mathrm{ml}$ of $T$. gondii STAg (5.57 fold; $P$-value $=0.0103$ ). Similar to Bcl-2, except the concentration $100 \mu \mathrm{g} / \mathrm{ml}$, increased expression was observed in low concentrations of T. gondii STAg (Fig 1B).

Cas-7 showed overexpression in all concentrations. The highest expression was observed in test wells sensed by 


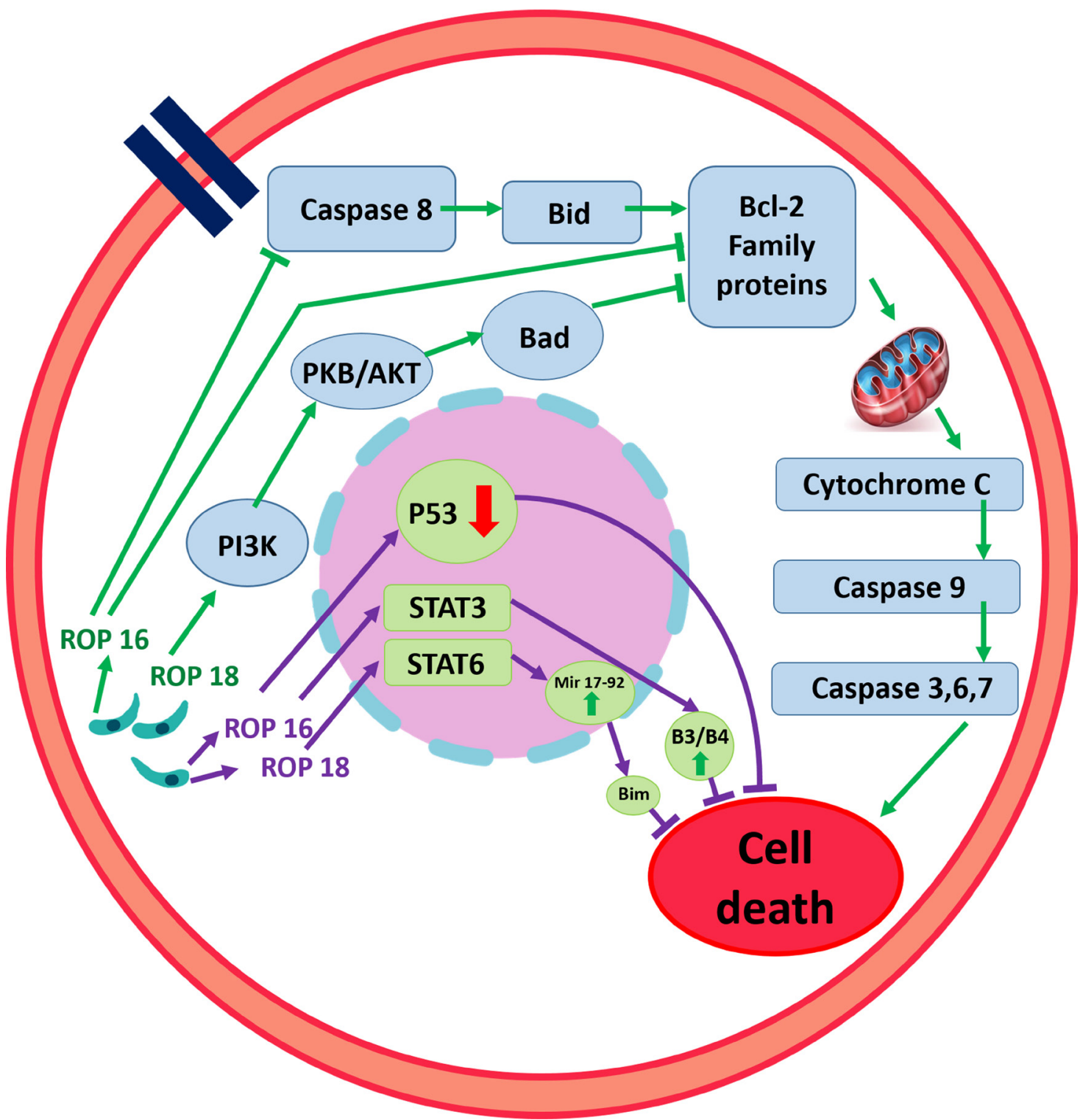

Fig 2. This schematic overview suggests signaling pathways of apoptosis. Apoptosis is induced through either the extrinsic pathway or intrinsic pathway. Toxoplasma gondii can prevent cell apoptosis via inhibition of cytochrome c release from the mitochondria into the cytosol, inhibition of cas-3, $-6,-7$, and -9 via blocking the mitochondrial pathway, and blocking cas- 8 by external pathways. The parasite may also suppress pro-apoptotic protein (Bax) and induce anti-apoptotic Bcl-2 proteins and is able to activate transcription factors STAT6 and STAT3-miR-17-92-Bim pathway to inhibit apoptosis. Some proteins of T. gondii such as ROP18 plays a role in Fas/ CD95-mediated apoptosis to inhibit this process. Similarly ROP16 phosphorylates STAT3 and STAT6 to inhibit cell death. Original (S. Nemati).

$100 \mu \mathrm{g}$ T. gondii STAg (19.53 fold; $P$-value $=0.002)$. In contrast to Bcl-2 and Mcl-1, a downregulation was seen in low concentrations $(P$-value $<0.0001)$ (Fig 1C).

The expression of cas-3 was suppressed in wells, which were sensed with concentrations 100,80 , and $40 \mu \mathrm{g} / \mathrm{ml}$ of T. gondii STAg, whereas it showed statistically significant downregulation in concentrations 10 and $20 \mu \mathrm{g} / \mathrm{ml}$ of $T$. gondii STAg with folds $4.53(P$-value $=0.01)$ and $2.21(P$-value $=0.028$ ), respectively (Fig. 1D). The expression of Bax (as pro-apoptotic protein) was suppressed in concentrations 100 to $20 \mu \mathrm{g} / \mathrm{ml}$ of $T$. gondii STAg, whereas it was slightly downregulated 1.42 fold $(P$-value $=0.0029)$ in concentration 10 $\mu \mathrm{g}$ (Fig. 1E). Interestingly, the expressions of cas- 8 and -9 were suppressed in all concentrations of $T$. gondii STAg.

\section{DISCUSSION}

At the beginning of the infection, Toxoplasma gondii provokes innate immunity system that leads to secretion of many different effectors. The host innate immunity is known to play a critical role in defensce against intracellular infection like toxoplasmosis (Sasai et al. 2018, Lima and Lodoen 2019). Followed by the innate immunity, the cell-mediated immune responses and released cytokines limit parasite replication and control toxoplasmosis (Sasai et al. 2018). Upon entrance of T. gondii, the host's cell em- 
Table 1. Primers used in this study.

\begin{tabular}{llll}
\hline Gene & Sense primer $\left(5^{\prime}-3^{\prime}\right)$ & Antisense primer $\left(5^{\prime}-3^{\prime}\right)$ & Refs \\
\hline Caspase 3 & CAGAACTGGACTGTGGCATTG & GCTTGTCGGCATACTGTTTCA & Xu et al. (2012) \\
Caspase 7 & GGAGAAAGCTCATGGCTGTGT & TCCCCTTGGCTGTGTTTTG & Tomita et al. (2003) \\
Caspase 8 & TCTGAAATCTGATAGAGCATGACC & AGACTGGATTTGCTGATTACCTAC & Ngobili et al. (2015) \\
Caspase 9 & GTCCTCAAACCTTCCTGGAAC & GCCCAAGCTCTTTTTCATCC & Ngobili et al. (2015) \\
Bax & ATGTTTCTGACGGCAACTTC & AGTCCAATGTCCAGCCCA & Buskaran et al. (2021) \\
Bcl-2 & AAGCCGGCGACGACTTCT & GGTGCCGGTTCAGGTACTCA & Laffon et al. (2001) \\
Mcl-1 & GGGCAGGATTGTGACTCTCATT & GATGCAGCTTTCTTGGTTTATGG & Rosato et al. (2007) \\
$\beta$-act & ATGTGGCCGAGGACTTTGATT & AGTGGGGTGGCTTTTAGGATG & Fu et al. (2010) \\
\hline
\end{tabular}

ploys immune responses, signalling pathways, transcriptional changes, and apoptosis mechanisms to prevent the development of the infection (Friedrich et al. 2017, Lima and Lodoen 2019). Besides the regulation of the immune responses, apoptosis acts as an effector mechanism of immune cells such as, natural killer (NK) cells and cytotoxic $\mathrm{T}$ lymphocytes, to eliminate infected target cells (Lieberman 2003, Opferman and Korsmeyer 2003).

To survive and replicate in host's cells, T. gondii employed different strategies to escape the immune system. This parasite manipulates the programmed cell death (apoptosis), as an innate response of cells, to enhance intracellular replication and long-term survival (Ammassari et al. 1996, Petersen et al. 2001, Zhu et al. 2019). Although the relationship between $T$. gondii and apoptosis pathways is controversial (Chu et al. 2017), the parasite probably decreases apoptosis in host cell via different pathways (Hisaeda et al. 1997, Nash et al. 1998, Goebel et al. 2001, Channon et al. 2002, Opferman and Korsmeyer 2003, Payne et al. 2003). Studies in both murine and human cell lines revealed the resistance of T. gondii against apoptosis (Nash et al. 1998, Goebel et al. 2001).

As a results, cas-3 and 9 were suppressed in $T$. gondii infected THP cells and cas-7 showed an increased expression at all concentrations. The expression of the Bax gene, as pro-apoptotic factors, was inhibited. In addition, the expression of Bcl-2 and Mcl-1, as anti-apoptotic factors, were increased upon treatment with $T$. gondii STAg. In the line of our study, the experimental evidence suggests that T. gondii inhibits apoptosis of host cells with manipulating of the apoptosis pathways. Goebel et al (2001) reported a downregulation of cas- 9 and cas-3, and blocked release of cytochrome C from mitochondria in HL-60 and U937 cell lines treated by $T$. gondii.

Furthermore, evidence indicated that the anti-apoptotic Mcl-1 and Bcl-2 increased after T. gondii infection (Goebel et al. 2001, Molestina et al. 2003). In addition, Hwang et al. (2010) suggested that T. gondii inhibited cas-3, but upregulated anti-apoptotic pathways via cytochrome C-mediated caspase activation. Although there are few data on the apoptosis and anti-apoptosis pathways induced by $T$. gondii in animal models, Kim and Denkers (2006) demonstrated that $T$. gondii provokes Gi-protein-dependent PI 3-kinase signaling to preclude apoptosis in host cell.

In the line of our findings, Payne et al (2003) suggested inhibition of cas- 8 activity by $T$. gondii in murine fibroblasts. Caspases are important effectors of programed cell death and their roles in apoptosis have been investigated
(Hotchkiss and Nicholson 2006). Caspase activation is stimulated by initiators cas- 8 and -9 that activate executioner cas-3. Activation of cas-3 and cas- 7 ultimately leads to apoptosis (Fig. 2). Lakhani et al. (2006) showed that although cas-3 and cas-7 are key mediators during the apoptosis process, cas-3 knockouted mice died prematurely, but the cas- 7 knockouted mice represented no phenotypic abnormalities. As well, cas-3-cas-7 knockouted mice suffered from early perinatal death.

Evidence points out the non-apoptotic functions of cas3 and cas- 7 in different cell processes like cell proliferation, cell cycle regulation, cell differentiation, and inflammation. Indeed, cas-3 and cas-7 seem to have independent functions during apoptosis (Lakhani et al. 2006, Lamkanfi et al. 2009, Lamkanfi and Kanneganti 2010). Actually, executioner caspases (cas-3 and/or cas-7) would be activated under inflammatory conditions, upon the activation of cas9 and -1 , as initiator caspases in the apoptosome and inflammasome complex, respectively (Hotchkiss and Nicholson 2006, Lamkanfi et al. 2009).

Unlike cas-3, cas-8, and cas-9, cas-7 was upregulated in our study. Cas-7 was suggested to be involved in inflammation. Lamkanfi et al. (2008) showed that upon the LPS stimulation, cas- 7 is activated by cas- 1 proposing an apoptosis-independent regulatory role for cas-7 in pro-inflammatory genes expression. In addition, it was suggested that macrophages are able to restrict Legionella pneumophila via NLRC4/IPAF inflammatory factors and cas-7 activation (Akhter et al. 2009).

These results are also supported by experimental studies that have shown that $T$. gondii or its proteins increase the expression levels of inflammasomes and inflammation (Witola et al. 2011, Chai and Shi 2014, Wang et al. 2019). However, the main differences between results most probably back to either the time points of experiments or the inducer. For example, Wang and Gao (2016) demonstrated that tachyzoites of $T$. gondii RH strain prevented the proliferation and induced apoptosis in HCC H7402 cells. In contrast, Wu et al. (2020) showed that rhoptry protein 16 (ROP16) inhibited programmed cell death in human $293 \mathrm{~T}$ cell. These studies may help interpretation of our results. Actually, in the present study we used T. gondii STAg, which contains a broad spectrum of proteins that unlike the alive tachyzoite, these proteins may manipulate apoptosis pathways via different manner.

Some studies also proposed that strains of $T$. gondii and time points affect the expression of genes which are involved in the apoptosis pathway. Angeloni et al. (2009) 
suggested that $T$. gondii strains affect the apoptosis. They showed that strain ME49, a moderately virulent strain, induced higher apoptosis index compared to RH strain, which is known as a highly virulent strain. Interestingly, our study supports this result that hypervirulent strains such as $\mathrm{RH}$, are probably able to inhibit programmed cell death upon the infection to increase their replication in host cell. In the line of our findings, Contreras-Ochoa et al. (2013) showed that $T$. gondii tachyzoite RH strain controlled the apoptosis pathways in host cells and except the early stage of infection, apoptosis decreased up to $24 \mathrm{~h}$ after infection.

Therefore, based on our results, it seems that $T$. gondii STAg probably induces inflammatory responses and suppresses apoptotic pathways in monocyte cell line, although alive tachyzoite may activate apoptosis pathways in host cells. However, we employed RH strain which is a hyper-virulent strain of T. gondii; therefore, the results of interaction between host cell apoptosis and $T$. gondii should be interpreted according to different strains of the parasite.
In conclusion, our results indicat that $T$. gondii STAg downregulated and suppressed apoptotic and upregulates anti-apoptotic pathways in human monocyte cell line. In addition, the upregulation of cas-7 in this study may indicate the role of $T$. gondii STAg in activation of inflammatory responses. However, the results of this and previous studies suggest that alive tachyzoite, STAg, and/or specific proteins derived from $T$. gondii may interplay with host cells via different manners.

Acknowledgment. The authors thank all members of the Foodborne and Waterborne Diseases Research Centre for their supports. This study was financially supported by the Research Institute for Gastroenterology and Liver Diseases, Shahid Beheshti University of Medical Sciences with grant number: RIGLD-1059. Ethics approval. All procedures performed in this study were in accordance with the ethical standards (IR.SBMU.RIGLD. REC.1398.035) released by Ethical Review Committee of the Research Institute for Gastroenterology and Liver Diseases, Shahid Beheshti University of Medical Sciences, Tehran, Iran.

\section{REFERENCES}

Aguirre A.A., Longcore T., Barbieri M., Dabritz H., Hill D., Klein P. N., Lepczyk C., Lilly E. L., Mcleod R., Milcarsky J., Murphy C.E., Su C., Vanwormer E., Yolken R., Sizemore G.C. 2019: The one health approach to toxoplasmosis: epidemiology, control, and prevention strategies. Ecohealth 16: 378-390.

Akhtar F., Bokhari S.R.A. 2021: Apoptosis. In: StatPearls Statpearls Publishing Llc., Treasure Island, NBK499821.

Akhter A., Gavrilin M.A., Frantz L., Washington S., Ditty C., Limoli D., Day C., Sarkar A., Newland C., Butchar J., Marsh C.B., Wewers M.D., Tridandapani S., Kanneganti T.D., Amer A. O. 2009: Caspase-7 activation by the Nlrc4/Ipaf inflammasome restricts Legionella pneumophila infection. PLoS. Pathog. 5: e1000361.

Ammassari A., Murri R., Cingolani A., Deluc A.A., AnTINORI A. 1996: Aids-associated cerebral toxoplasmosis: an update on diagnosis and treatment. Curr. Top. Microbiol. Immunol. 219: 209-222.

Angeloni M.B., Silva N.M., Castro A.S., Gomes A.O., Silva D.A., Mineo J. R., Ferro E.A. 2009: Apoptosis and $\mathrm{S}$ phase of the cell cycle in bewo trophoblastic and hela cells are differentially modulated by Toxoplasma gondii strain types. Placenta 30: 785-791.

Boatright K.M., Renatus M., Scott F.L., Sperandio S., Shin H., Pedersen I.M., Ricci J.E., Edris W.A., Sutherlin D.P., Green D.R., Salvesen G.S. 2003: A unified model for apical caspase activation. Mol. Cell. 11: 529-541.

Buskaran K., Bullo S., Hussein M.Z, Masarudin M.J, Mohd Moklas M.A., FAKurazi S. 2021: Anticancer molecular mechanism of protocatechuic acid loaded on folate coated functionalized graphene oxide nanocomposite delivery system in human hepatocellular carcinoma. Materials (Basel) 14: 817.

Chai J., Shi Y. 2014: Apoptosome and inflammasome: conserved machineries for caspase activation. Nat. Sci. Rev. 1: 101-118.

Channon J.Y., Miselis K.A., Minns L.A., Dutta C., Kasper L.H. 2002: Toxoplasma gondii induces granulocyte colonystimulating factor and granulocyte-macrophage colonystimulating factor secretion by human fibroblasts: implications for neutrophil apoptosis. Infect. Immun. 70: 6048-6057.

Cheng E.H., Wei M.C., Weiler S., Flavell R.A., Mak T.W., Lindsten T., Korsmeyer S.J. 2001: Bcl-2, Bcl-X(L) sequester Bh3 domain-only molecules preventing Bax- and Bak-mediated mitochondrial apoptosis. Mol. Cell. 8: 705-711.
Chu J.Q., Jing K.P., Gao X., Li P., Huang R., Niu Y.R., Yan S.Q., Kong J.C., Yu C.Y., Shi G., FAn Y.M., Lee Y.H., Zhou Y., QuAN J.H. 2017: Toxoplasma gondii induces autophagy and apoptosis in human umbilical cord mesenchymal stem cells via downregulation of Mcl-1. Cell. Cycle 16: 477-486.

Contreras-Ochoa C.O., Lagunas-Martínez A., BelkindGerson J., Díaz-Chávez J., Correa D. 2013: Toxoplasma gondii invasion and replication within neonate mouse astrocytes and changes in apoptosis related molecules. Exp. Parasitol. 134: 256-265.

D'ARCY M.S. 2019: Cell death: a review of the major forms of apoptosis, necrosis and autophagy. Cell. Biol. Int. 43: 582-592.

Elmore S. 2007: Apoptosis: a review of programmed cell death. Toxicol. Pathol. 35: 495-516.

Friedrich A., Pechstein J., Berens C., Lührmann A. 2017: Modulation of host cell apoptotic pathways by intracellular pathogens. Curr. Opin. Microbiol. 35: 88-99.

Fu J., Bian L., Zhao L., Dong Z., Gao X., Luan H., Sun Y., Song H. 2010: Identification of genes for normalization of quantitative real-time PCR data in ovarian tissues. Acta Biochim. Biophys. Sin. 42: 568-574.

Goebel S., Gross U., Lüder C.G. 2001: Inhibition of host cell apoptosis by Toxoplasma gondii is accompanied by reduced activation of the caspase cascade and alterations of poly (adpribose) polymerase expression. J. Cell. Sci. 114: 3495-3505.

Green D., Knight R., Melino G., Finazzi-Agro A., Orrenius S. 2004: Ten years of publication in cell death. Cell Death Differ. 11: $2-3$.

Hisaeda H., Sakai T., Ishikawa H., Maekawa Y., Yasutomo K., Good R.A., Himeno K. 1997: Heat shock protein 65 induced by gammadelta $\mathrm{T}$ cells prevents apoptosis of macrophages and contributes to host defense in mice infected with Toxoplasma gondii. J. Immunol. 159: 2375-2381.

Hotchkiss R.S., Nicholson D.W. 2006: Apoptosis and caspases regulate death and inflammation in sepsis. Nat. Rev. Immunol. 6: 813-822.

Hwang I.Y., Quan J.H., Ahn M.H., Ahmed H.A., Cha G.H., Shin D.W., LeE Y.H. 2010: Toxoplasma gondii infection inhibits the mitochondrial apoptosis through induction of Bcl-2 and Hsp70. Parasitol. Res. 107: 1313-1321.

Jin Z., El-Deiry W.S. 2005: Overview of cell death signaling pathways. Cancer. Biol. Therap. 4: 147-171. 
Kim L., Denkers E.Y. 2006: Toxoplasma gondii triggers gidependent Pi 3-kinase signaling required for inhibition of host cell apoptosis. J. Cell. Sci. 119: 2119-2126.

Laffon B., Pásaro E., Méndez J. 2001: Effects of styrene7,8-oxide over P53, P21, Bcl-2 and Bax expression in human lymphocyte cultures. Mutagenesis 16: 127-132.

Lakhani S.A., Masud A., Kuida K., Porter G.A. Jr., Booth C.J., Mehal W.Z., Inayat I., Flavell R.A. 2006: Caspases 3 and 7: key mediators of mitochondrial events of apoptosis. Science 311: 847-851.

Lamkanfi M., Kanneganti T.D. 2010: Caspase-7: a protease involved in apoptosis and inflammation. Int. J. Biochem. Cell. Biol. 42: 21-24.

Lamkanfi M., Kanneganti T.D., Van Damme P., Vanden Berghe T., Vanoverberghe I., Vandekerckhove J., Vandenabeele P., Gevaert K., NúÑez G. 2008: Targeted peptidecentric proteomics reveals caspase-7 as a substrate of the caspase-1 inflammasomes. Mol. Cell. Proteomics 7: 2350-2363.

Lamkanfi M., Moreira L.O., Makena P., Spierings D.C., Boyd K., Murray P.J., Green D.R., Kanneganti T.D. 2009: Caspase-7 deficiency protects from endotoxin-induced lymphocyte apoptosis and improves survival. Blood 113: 2742-2745.

Li M., Wang H., LiU J., Hao P., Ma L., LiU Q. 2016: The apoptotic role of metacaspase in Toxoplasma gondii. Front. Microbiol. 6: 1560.

Lieberman J. 2003: The abcs of granule-mediated cytotoxicity: new weapons in the arsenal. Nat. Rev. Immunol. 3: 361-370.

Lima T.S., Lodoen M.B. 2019: Mechanisms of human innate immune evasion by Toxoplasma gondii. Front. Cell. Infect. Microbiol. 9: 103.

Lourido S. 2019: Toxoplasma gondii. Trends Parasitol. 35: 944-945.

LÜDER C.G., Gross U., LOPES M.F. 2001: Intracellular protozoan parasites and apoptosis: diverse strategies to modulate parasitehost interactions. Trends Parasitol. 17: 480-486.

Mammari N., Halabi M.A., Yaacoub S., Chlala H., Dardé M.L., Courtioux B. 2019: Toxoplasma gondii modulates the host cell responses: an overview of apoptosis pathways. Biomed. Res. Int. 2019: 6152489.

Molestina R.E., Payne T.M., Coppens I., Sinai A.P. 2003: Activation of NF- $\mathrm{kB}$ by Toxoplasma gondii correlates with increased expression of antiapoptotic genes and localization of phosphorylated ikb to the parasitophorous vacuole membrane. J. Cell. Sci. 116: 4359-4371

Nash P.B., Purner M.B., Leon R.P., Clarke P., Duke R.C., CuRIEL T.J. 1998: Toxoplasma gondii-infected cells are resistant to multiple inducers of apoptosis. J. Immunol. 160: 1824-1830.

Ngobili T.A., Shah H., Park J.P., Kwist K.W., Inskeep B., Burg K.J., Bоотн B.W. 2015: Remodeling of tannic acid crosslinked collagen type $\mathrm{i}$ induces apoptosis in ER+ breast cancer cells. Anticancer Res. 35: 1285-1290.

OBEnG E. 2021: Apoptosis (programmed cell death) and its signals - a review. Braz. J. Biol. 81: 1133-1143.

Opferman J.T., Korsmeyer S.J. 2003: Apoptosis in the development and maintenance of the immune system. Nat. Immunol. 4: 410-415.

Pappas G., Roussos N., Falagas M.E. 2009: Toxoplasmosis snapshots: global status of Toxoplasma gondii seroprevalence and implications for pregnancy and congenital toxoplasmosis. Int. J. Parasitol. 39: 1385-1394.

Payne T.M., Molestina R.E., Sinai A.P. 2003: Inhibition of caspase activation and a requirement for $\mathrm{nf}-\mathrm{kb}$ function in the
Toxoplasma gondii-mediated blockade of host apoptosis. J. Cell. Sci. 116: 4345-4358.

Pazoki H., Mohammad Rahimi H., Mirjalali H., Niyyati M., Mosaffa N., Seyed Tabaei S.J., Shahrokh S., Asadzadeh Aghdaei H., Zali M. R. 2021: Soluble total antigen derived from Toxoplasma gondii tachyzoites increased the expression levels of nlrp1, nlrp3, nlrc4, aim2, and the release of mature form of il1 $\beta$, but downregulated the expression of $i 11 \beta$ and ills genes in thp-1 cell line. Microb. Pathog. 105072.

Petersen E., Pollak A., Reiter-Owona I. 2001: Recent trends in research on congenital toxoplasmosis. Int. J. Parasitol. 31: 115-144.

Rosato R.R., Almenara J.A., Kolla S.S., Maggio S.C., Coe S., Giménez M.S., Dent P., Grant S. 2007: Mechanism and functional role of xiap and mcl-1 down-regulation in flavopiridol/ vorinostat antileukemic interactions. Mol. Cancer. Therap. 6: 692-702.

Sasai M., Pradipta A., Yamamoto M. 2018: Host immune responses to Toxoplasma gondii. Int. Immunol. 30: 113-119.

Tenter A.M., Heckeroth A.R., Weiss L.M. 2000: Toxoplasma gondii: from animals to humans. Int. J. Parasitol. 30: 1217-1258.

Tomita Y., Bilim V., Hara N., Kasahara T., Takahashi K. 2003: Role of irf-1 and caspase-7 in ifn-gamma enhancement of fas-mediated apoptosis in achn renal cell carcinoma cells. Int. J. Cancer 104: 400-408.

Toulah F.H., Sayed Al-Ahl S.A., Amin D.M., Hamouda M.H. 2011: Toxoplasma gondii: ultrastructure study of the entry of tachyzoites into mammalian cells. Saudi. J. Biol. Sci. 18: $151-156$.

WANG G., GaO M. 2016: Influence of Toxoplasma gondii on in vitro proliferation and apoptosis of hepatoma carcinoma h7402 cell. Asian Pac. J. Trop. Med. 9: 63-66.

Wang Y., Cirelli K.M., Barros P., Sangaré L.O., Butty V., Hassan M.A., Pesavento P., Mete A., Saeij J. 2019: Three Toxoplasma gondii dense granule proteins are required for induction of lewis rat macrophage pyroptosis. mBio. 10: e0238818.

Witola W.H., Mui E., Hargrave A., Liu S., Hypolite M., Montpetit A., Cavailles P., Bisanz C., CesbronDelauw M,F., Fournié G.J., Mcleod R. 2011: NALP1 influences susceptibility to human congenital toxoplasmosis, proinflammatory cytokine response, and fate of Toxoplasma gondii-infected monocytic cells. Infect. Immun. 79: 756-766.

Wu L., Wu L., Xi C., Liu Y., Jiang X., Chen S., Cao J. 2020: Toxoplasma gondii rhoptry protein 16 (ROP16) modifies apoptosis in human 293 T cells. J. Nanosci. Nanotechnol. 20: 24-30.

Xiao J., Yolken R.H. 2015: Strain hypothesis of Toxoplasma gondii infection on the outcome of human diseases. Acta Physiol. 213: 828-845.

Xu T., Wang N.S., Fu L.L., Ye C.Y., Yu S.Q., Mei C.L. 2012: Celecoxib inhibits growth of human autosomal dominant polycystic kidney cyst-lining epithelial cells through the VEGF/ Raf/MAPK/ERK signaling pathway. Mol. Biol. Rep. 39: $7743-$ 7753.

Yanumula A., Cusick J.K. (Eds.) 2021: Biochemistry, Extrinsic Pathway of Apoptosis. In: StatPearls. Statpearls Publishing Llc., Treasure Island, NBK560811.

Zhu W., Li J., Pappoe F., Shen J., Yu, L. 2019: Strategies developed by Toxoplasma gondii to survive in the host. Front. Microbiol. 10: 899-899.

Cite this article as: Rahimi H.M., Nemati S., Alavifard H., Baghaei K., Mirjalali H., Zali M.R. 2021: Soluble total antigen derived from Toxoplasma gondii RH strain prevents apoptosis, but induces anti-apoptosis in human monocyte cell line. Folia Parasitol. 68: 026 . 